\title{
Performance of an Automated Versus a Manual Whole-Body Magnetic Resonance Imaging Workflow
}

\author{
Stocker, Daniel ; Finkenstaedt, Tim ; Kuehn, Bernd ; Nanz, Daniel ; Klarhoefer, Markus ; \\ Guggenberger, Roman ; Andreisek, Gustav ; Kiefer, Berthold ; Reiner, Caecilia S
}

\begin{abstract}
OBJECTIVES: The aim of this study was to evaluate the performance of an automated workflow for whole-body magnetic resonance imaging (WB-MRI), which reduces user interaction compared with the manual WB-MRI workflow. MATERIALS AND METHODS: This prospective study was approved by the local ethics committee. Twenty patients underwent WB-MRI for myopathy evaluation on a 3 T MRI scanner. Ten patients ( 7 women; age, $52 \pm 13$ years; body weight, $69.9 \pm 13.3 \mathrm{~kg}$; height, $173 \pm 9.3 \mathrm{~cm}$; body mass index, $23.2 \pm 3.0)$ were examined with a prototypical automated WB-MRI workflow, which automatically segments the whole body, and 10 patients ( 6 women; age, $35.9 \pm 12.4$ years; body weight, $72 \pm 21 \mathrm{~kg}$; height, $169.2 \pm 10.4 \mathrm{~cm}$; body mass index, $24.9 \pm 5.6)$ with a manual scan. Overall image quality (IQ; 5-point scale: 5, excellent; 1 , poor) and coverage of the study volume were assessed by 2 readers for each sequence (coronal T2-weighted turbo inversion recovery magnitude [TIRM] and axial contrast-enhanced T1-weighted [ce-T1w] gradient dual-echo sequence). Interreader agreement was evaluated with intraclass correlation coefficients. Examination time, number of user interactions, and MR technicians' acceptance rating (1, highest; 10, lowest) was compared between both groups. RESULTS: Total examination time was significantly shorter for automated WB-MRI workflow versus manual WB-MRI workflow $(30.0 \pm 4.2$ vs $41.5 \pm 3.4$ minutes, $\mathrm{P}<0.0001)$ with significantly shorter planning time $(2.5 \pm 0.8$ vs $14.0 \pm 7.0$ minutes, $\mathrm{P}<0.0001)$. Planning took $8 \%$ of the total examination time with automated versus $34 \%$ with manual WB-MRI workflow $(\mathrm{P}<0.0001)$. The number of user interactions with automated WB-MRI workflow was significantly lower compared with manual WB-MRI workflow $(10.2 \pm 4.4$ vs $48.2 \pm 17.2, \mathrm{P}<0.0001)$. Planning efforts were rated significantly lower by the MR technicians for the automated WB-MRI workflow than for the manual WB-MRI workflow $(2.20 \pm$ 0.92 vs $4.80 \pm 2.39$, respectively; $\mathrm{P}=0.005)$. Overall IQ was similar between automated and manual WB-MRI workflow (TIRM: $4.00 \pm 0.94$ vs $3.45 \pm 1.19, \mathrm{P}=0.264$; ce-T1w: $4.20 \pm 0.88$ vs $4.55 \pm .55$, $\mathrm{P}=0.423)$. Interreader agreement for overall IQ was excellent for TIRM and ce-T1w with an intraclass correlation coefficient of 0.95 (95\% confidence interval, 0.86-0.98) and 0.88 (95\% confidence interval, $0.70-0.95)$. Incomplete coverage of the thoracic compartment in the ce- $\mathrm{T} 1 \mathrm{w}$ sequence occurred more often in the automated WB-MRI workflow $(\mathrm{P}=0.008)$ for reader 2 . No other significant differences in the study volume coverage were found. CONCLUSIONS: In conclusion, the automated WB-MRI scanner workflow showed a significant reduction of the examination time and the user interaction compared with the manual WB-MRI workflow. Image quality and the coverage of the study volume were comparable in both groups.
\end{abstract}

DOI: https://doi.org/10.1097/RLI.0000000000000471 


\section{Published Version}

Originally published at:

Stocker, Daniel; Finkenstaedt, Tim; Kuehn, Bernd; Nanz, Daniel; Klarhoefer, Markus; Guggenberger, Roman; Andreisek, Gustav; Kiefer, Berthold; Reiner, Caecilia S (2018). Performance of an Automated Versus a Manual Whole-Body Magnetic Resonance Imaging Workflow. Investigative Radiology, 53(8):463471.

DOI: https://doi.org/10.1097/RLI.0000000000000471 


\title{
Performance of an Automated Versus a Manual Whole-Body Magnetic Resonance Imaging Workflow
}

\author{
Daniel Stocker, MD, *广 Tim Finkenstaedt, MD, *广 Bernd Kuehn, PhD, $\neq$ Daniel Nanz, PhD, *† \\ Markus Klarhoefer, PhD, § Roman Guggenberger, MD, *广 Gustav Andreisek, MD, $†$ - \\ Berthold Kiefer, PhD, $\neq$ and Caecilia S. Reiner, MD*广
}

Objectives: The aim of this study was to evaluate the performance of an automated workflow for whole-body magnetic resonance imaging (WB-MRI), which reduces user interaction compared with the manual WB-MRI workflow.

Materials and Methods: This prospective study was approved by the local ethics committee. Twenty patients underwent WB-MRI for myopathy evaluation on a 3 T MRI scanner. Ten patients ( 7 women; age, $52 \pm 13$ years; body weight, $69.9 \pm 13.3 \mathrm{~kg}$; height, $173 \pm 9.3 \mathrm{~cm}$; body mass index, $23.2 \pm 3.0$ ) were examined with a prototypical automated WB-MRI workflow, which automatically segments the whole body, and 10 patients ( 6 women; age, $35.9 \pm 12.4$ years; body weight, $72 \pm 21 \mathrm{~kg}$; height, $169.2 \pm 10.4 \mathrm{~cm}$; body mass index, $24.9 \pm 5.6)$ with a manual scan. Overall image quality (IQ; 5-point scale: 5 , excellent; 1, poor) and coverage of the study volume were assessed by 2 readers for each sequence (coronal T2-weighted turbo inversion recovery magnitude [TIRM] and axial contrast-enhanced T1-weighted [ce-T1w] gradient dual-echo sequence). Interreader agreement was evaluated with intraclass correlation coefficients. Examination time, number of user interactions, and MR technicians' acceptance rating (1, highest; 10 , lowest) was compared between both groups.

Results: Total examination time was significantly shorter for automated WB-MRI workflow versus manual WB-MRI workflow $(30.0 \pm 4.2$ vs $41.5 \pm 3.4$ minutes, $P<0.0001)$ with significantly shorter planning time $(2.5 \pm 0.8$ vs $14.0 \pm 7.0$ minutes, $P<0.0001)$. Planning took $8 \%$ of the total examination time with automated versus $34 \%$ with manual WB-MRI workflow $P<0.0001)$. The number of user interactions with automated WB-MRI workflow was significantly lower compared with manual WB-MRI workflow $(10.2 \pm 4.4$ vs $48.2 \pm 17.2, P<0.0001)$. Planning efforts were rated significantly lower by the MR technicians for the automated WB-MRI workflow than for the manual WB-MRI workflow ( $2.20 \pm 0.92$ vs $4.80 \pm 2.39$, respectively; $P=0.005)$. Overall IQ was similar between automated and manual WB-MRI workflow (TIRM: $4.00 \pm 0.94$ vs $3.45 \pm 1.19, P=0.264$; ce-T1w: $4.20 \pm 0.88$ vs $4.55 \pm .55, P=0.423)$. Interreader agreement for overall IQ was excellent for TIRM and ce-T1w with an intraclass correlation coefficient of 0.95 (95\% confidence interval, $0.86-0.98)$ and 0.88 (95\% confidence interval, $0.70-0.95)$. Incomplete coverage of the thoracic compartment in the ce- $\mathrm{T} 1 \mathrm{w}$ sequence occurred more often in the automated WB-MRI workflow $(P=0.008)$ for reader 2. No other significant differences in the study volume coverage were found. Conclusions: In conclusion, the automated WB-MRI scanner workflow showed a significant reduction of the examination time and the user interaction compared

Received for publication January 24, 2018; and accepted for publication, after revision, February 22, 2018.

From the *Institute of Diagnostic and Interventional Radiology, University Hospital Zürich; †University of Zürich, Zürich, Switzerland; †Oncology Application Predevelopment, Siemens Healthcare GmbH, Erlangen, Germany; §Siemens Healthcare AG, Zürich; and \|Radiologie, Spital Thurgau, Kantonsspital Munsterlingen, Münsterlingen, Switzerland.

Conflict of Interest: Bernd Kuehn, $\mathrm{PhD}$, and Berthold Kiefer, $\mathrm{PhD}$, are employees of Siemens Healthcare GmbH, Erlangen, Germany. Markus Klarhöfer, PhD, is an employee of Siemens Healthcare AG, Zürich. All other authors declare that they have no conflict of interest.

Correspondence to: Caecilia S. Reiner, MD, Institute of Interventional and Diagnostic Radiology, University Hospital Zurich and University of Zurich, Raemistrasse 100, 8091 Zürich, Switzerland. E-mail: caecilia.reiner@gmx.at.

Copyright (c) 2018 Wolters Kluwer Health, Inc. All rights reserved.

ISSN: 0020-9996/18/0000-0000

DOI: $10.1097 /$ RLI.0000000000000471 with the manual WB-MRI workflow. Image quality and the coverage of the study volume were comparable in both groups.

Key Words: WB-MRI, automated MRI workflow, image quality, examination time

(Invest Radiol 2018;00: 00-00)

diopathic inflammatory myopathies (IIMs) are rare autoimmune disorders most commonly subclassified in dermatomyositis, polymyositis, necrotizing autoimmune myopathy, and nonspecific myositis. ${ }^{1-4}$ Although diagnosis of IIM is based on a variety of clinical presentations, laboratory parameters, and muscle biopsy, whole-body magnetic resonance imaging (WB-MRI) has been shown to be a promising diagnostic tool, ${ }^{5-9}$ for example, to assess the extent of disease or localize the ideal location for a diagnostic muscle biopsy.

However, WB-MRI is a time-consuming examination with a total examination time typically ranging from 43 to 56 minutes. ${ }^{8,10,11}$ Multiple planning steps have to be performed by the technician on the scanner, and image acquisition itself takes more time than for single body region examinations, if the whole body needs to be covered in 1 examination. Due to efficiency aspects, patients' acceptance, and comfort as well as potential negative effects of patients' discomfort on image quality (IQ), examination time should be kept as short as possible.

This time constraint in mind, Filli et al ${ }^{10}$ tried to shorten the WB-MRI protocol for the detection of IIM by dropping imaging of the trunk with similar accuracy compared with standard WB-MRI. Although IIM in adults most frequently affects the upper or lower limbs, ${ }^{12,13}$ in young patients with juvenile inflammatory myopathies, the abdominal wall is affected in more than $50 \%{ }^{14}$ Beside efforts to reduce the actual scan time by omitting specific imaging sequences or designing faster sequences, a recent focus has been the automation of the MRI workflow: automated scanner workflows can allow a reduction of the total examination time and the number of planning steps and user interactions during the MR scan. A similar automated scanner workflow was previously investigated for MRI examinations of the head and led to a significant reduction of the total examination time in comparison to standard nonautomated MRI examinations. ${ }^{15}$ The potential of an automated scanner workflow with fewer planning steps and user interactions may be particularly large for WB-MRI, where typically a large number of planning steps are needed.

Therefore, the purpose of this study was to evaluate the performance of an automated WB-MRI scanner workflow reducing user interaction compared with the manual MRI workflow for myopathy evaluation.

\section{MATERIALS AND METHODS}

This was a prospective study approved by the local ethics committee. Written informed consent was obtained from each patient. The study was part of a larger, still ongoing study where the influence of examination time on patient comfort during an MRI examination was evaluated.

\section{Study Population}

A total of 30 patients ( 15 women, 15 men; mean age, 46 years; range, $21-70$ years; mean body mass index [BMI], $26.4 \mathrm{~kg} / \mathrm{m}^{2}$; range, 
$18.4-40.4 \mathrm{~kg} / \mathrm{m}^{2}$ ) were scheduled for clinically indicated WB-MRI for myopathy evaluation between September 2016 and December 2016 at our institute. First, 11 consecutive patients were examined with the whole-body protocol with manual planning, then 19 were examined with the automated whole-body protocol. Exclusion criteria were (1) age younger than 18 years, (2) contraindications for MRI scanning, and (3) contraindications for MR contrast media. Ten patients had to be excluded from the analysis: 6 due to technical problems (failure to start the sequence or changes of sequence parameters due to exceeded specific absorption rate limits) and change of protocol during the examination ( 5 with the automated WB-MRI, 1 with the manual WB-MRI), 3 due to incomplete MRI examination (lower legs were not examined due to individual planning mistakes by the technician, all with the automated WB-MRI), and 1 due to termination of the examination by the patient (with the automated WB-MRI).

The final study population consisted of 20 patients (13 women; 7 men; mean age, 44 years; range, 21-67 years; mean BMI, $24.0 \mathrm{~kg} / \mathrm{m}^{2}$; range, $18.4-38.9 \mathrm{~kg} / \mathrm{m}^{2}$ ). Ten patients were scanned in group A with an automated WB-MRI workflow (7 women; 3 men; mean age, 52 years; range, 28-67 years; mean BMI, $23.2 \mathrm{~kg} / \mathrm{m}^{2}$; range, $18.4-27.6 \mathrm{~kg} / \mathrm{m}^{2}$ ) and 10 in group B with a manual WB-MRI workflow (6 women; 4 men; mean age, 36 years; range, 21-52 years; mean BMI, $24.9 \mathrm{~kg} / \mathrm{m}^{2}$; range, $18.9-38.9 \mathrm{~kg} / \mathrm{m}^{2}$ ).

\section{Magnetic Resonance Imaging}

All images were acquired on a 3 T MRI scanner (MAGNETOM Skyra; Siemens Healthcare, Erlangen, Germany) using a coil set covering the whole body, including a flexible 20-channel head/neck coil, two 18-channel body coils, a 32-channel spine coil, and a 36-channel peripheral coil.

Patients in group A underwent the automated WB-MRI workflow including a coronal fast low-resolution whole-body scout acquired during moving table with automated executed breath-hold order (preset maximum breath-hold of 12 seconds; repetition time [TR], 2.56 milliseconds; echo time [TE], 1.4 milliseconds; field of view in head feet direction [FoV], $2400 \mathrm{~mm}$; slice thickness [ST], $5 \mathrm{~mm}$; voxel size, $5 \times 5 \times 5 \mathrm{~mm}^{3}$ ), 6 to 7 stacks (neck, thoracic, abdominal, pelvic, and upper and lower leg region) of a coronal 2-dimensional turbo inversion recovery magnitude sequence (TIRM) (respiratory triggering in the thoracic and abdominal regions; TR, 2200 milliseconds; TE, 260 milliseconds; flip angle [FA], 120 degrees; FoV, 500 mm; ST, $4 \mathrm{~mm}$; voxel size, $1.6 \times 1.6 \times 4 \mathrm{~mm}^{3}$ ), and 6 to 7 stacks (neck, thoracic, abdominal, pelvic, and upper and lower leg region) of an axial contrast-enhanced T1-weighted (ce-T1w) 3-dimensional (3D) dual-gradient echo sequence (volume interpolated breath-hold examination, VIBE) with Dixon-based fat-water separation (preset maximum breath-hold of 12 seconds in the thoracic and abdominal regions; TR, 4.25 milliseconds; TE1, 1.37 milliseconds; TE2, 2.55 milliseconds; FA, 16 degrees; FoV, $500 \mathrm{~mm}$; ST, $3 \mathrm{~mm}$; voxel size, $1.6 \times 1.6 \times 3 \mathrm{~mm}^{3}$ ).

Patients in group B underwent manually planned whole-body scanning with 6 stacks of each sequence type (neck, thoracic, abdominal, pelvic, and upper and lower leg region): coronal half-Fourier acquisition single-shot turbo spin echo (HASTE) localizer (TR, 1500 milliseconds; TE, 84 milliseconds; FA, 150 degrees; FoV, 500 mm; ST, 6 mm; voxel size, $1.6 \times 1.6 \times 6 \mathrm{~mm}^{3}$ ), coronal 2-dimensional TIRM (respiratory triggering in the thoracic and abdominal regions; TR, 2200 milliseconds; TE, 260 milliseconds; FA, 120 degrees; FoV, 500 mm; ST, 4 mm; voxel size, $\left.1.6 \times 1.6 \times 4 \mathrm{~mm}^{3}\right)$, and axial ce-T1w 3D dual gradient-echo VIBE with Dixon-based fat-water separation (preset maximum breath-hold of $12 \mathrm{sec}-$ onds in the thoracic and abdominal regions; TR, 4.91 milliseconds; TE1, 1.29 milliseconds; TE2, 2.52; FA, 16 degrees; FoV, 500 mm; ST, 3 mm; voxel size, $1.6 \times 1.6 \times 3 \mathrm{~mm}^{3}$ ).

In the automated WB-MRI workflow, the minimum and maximum head-feet coverage of 1 stack was predefined for each sequence.
Depending on the height of the patient and - in case of ce-T1w sequence - the patient's breath-hold capacity the whole head-feet coverage was automatically divided into 6 to 7 stacks. In the manual workflow, there was no maximum head-feet range for 1 stack, but the range of each of the 6 stacks was adjusted to the body region by the technician.

For contrast-enhanced sequences Gadoteric acid (Gd-DOTA) at a dose of $0.1 \mathrm{mmol}$ per kilogram body weight was injected followed by a saline-flush of $30 \mathrm{~mL}$.

\section{Planning Procedure}

\section{Automated WB-MRI Scanner Workflow}

First, a coronal fast low-resolution whole-body scout sequence was acquired during moving table from head to toe with breath-hold in the thoracic and abdominal region. On these images, the target study volume was automatically identified by an algorithm that detected anatomical landmarks (eg, lung apex, liver apex, and iliac bone). Based on the landmarks, the automatic workflow divided the entire study volume into separate anatomic regions (neck, thoracic, abdominal, pelvic, and leg region) that were transparent to the operator by planning boxes overlaid on the scout images (Fig. 1, left). If necessary, the boxes could then be manually optimized, if required specifically for each sequence. The technicians were instructed to cover the whole body from the lower neck to the ankle. After confirming the planning boxes, the coronal TIRM sequence was executed without any user interaction. Coil selection and respiratory triggering were controlled automatically by the scanner. After finishing the TIRM acquisition, a dialog window indicated that the examination was paused and prompted the operator to inject contrast media. Next, the technician confirmed to start the axial ce-T1w sequence. No further adaptions were required. Typically, the optimized study volume of the TIRM sequence was automatically copied, and again, coil selections were controlled automatically by the scanner. Breath-hold commands were performed automatically for the thoracic and abdominal regions (preset maximum duration of 12 seconds), whereas all other regions are scanned without breath-hold commands. The workflow adopts the protocol to meet the requested breath-hold duration. The separate imaging stacks were merged by the scanner to create a single coronal TIRM and a single axial ce-T1w whole-body image stack, respectively.

An example of the fast low-resolution whole-body scout and the planning boxes is shown in Figure 1.

\section{Manual WB-MRI Workflow}

First, separate coronal localizer sequences for the neck, thoracic, abdominal, pelvic, and upper and lower leg regions were acquired. The study volume was set by placing planning boxes for each localizer sequence separately and adjusted in each direction by manual input of the FoV and slice count. Similar, several coronal TIRM sequences were manually planned to cover the anatomical region from the neck to the lower legs. For respiratory triggering of the thoracic and abdominal region, a small box was manually placed on the liver dome in the localizer sequence. After finishing the TIRM sequence, a dialog window indicated that the examination was paused, and contrast media injection was initiated by the technician. Planning of the ce-T1w sequence was performed during acquisition of the TIRM sequences. For the ce-T1w sequence, several planning boxes were placed and adjusted in each direction in a fused vision of the coronal localizer sequences (Fig. 1, right). Again, the FoV and slice count were set by manual input for each anatomical region. For the ce-T1w sequence, breath-hold commands were performed automatically for thoracic and abdominal regions; all other regions were scanned without breath-hold. Coil selection was performed automatically by the scanner for both the TIRM and the ceT1w sequence. The separate study volumes were merged by the scanner to create 1 coronal TIRM and axial ce-T1w sequence, respectively. 


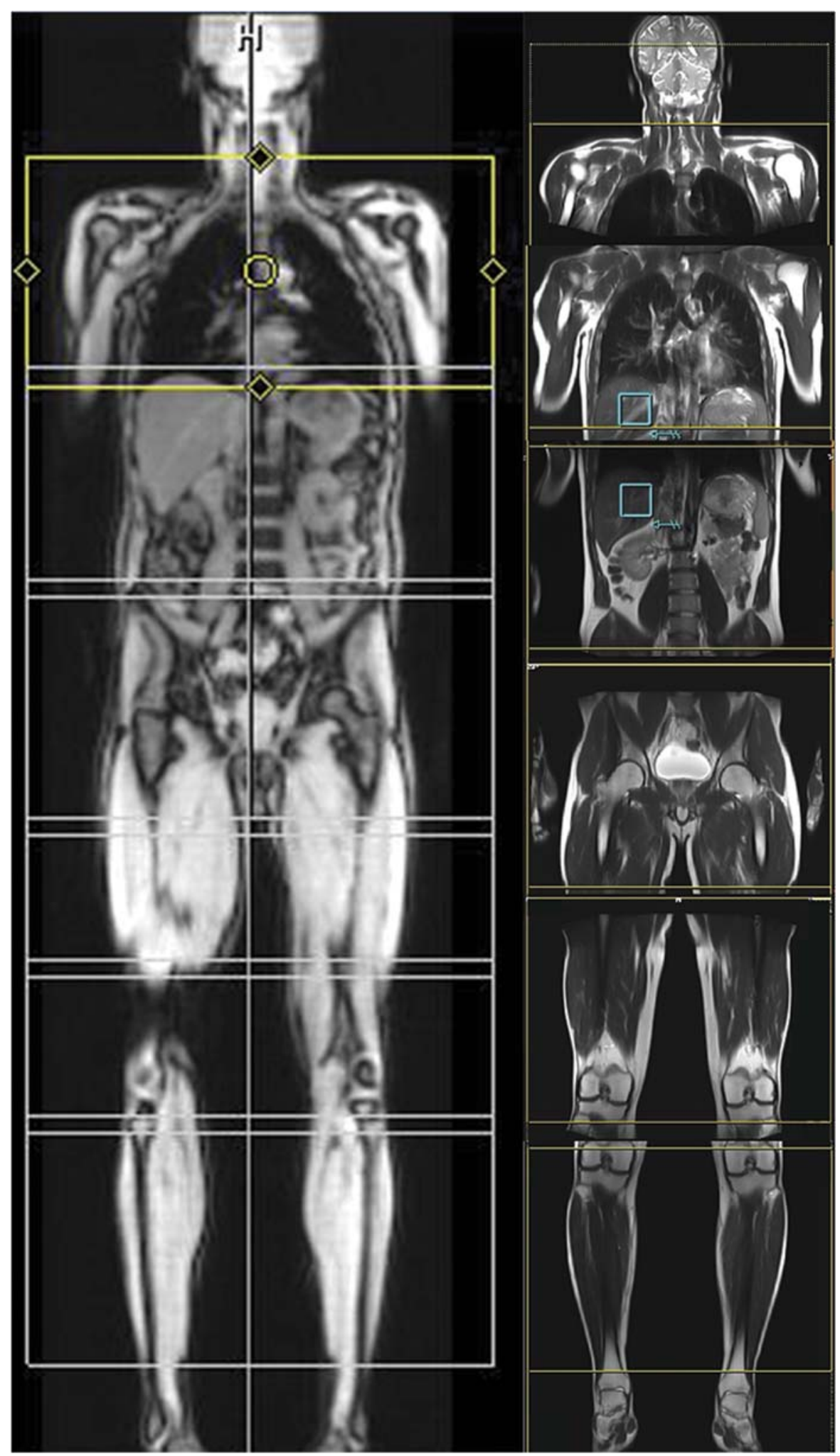

FIGURE 1. Example of the planning process on coronal scout sequences. Left, Planning process of automated WB-MRI scanner workflow with 1 fast low-resolution moving table whole-body localizer and 1 planning area for the whole body. Right, Planning process of manual WB-MRI scanner workflow with 6 separately acquired and displayed localizer sequences and separate planning areas for thorax, abdomen, pelvis, and upper and lower legs. Selective scans in the box manually placed in the liver parenchyma allow phase-based registration of diaphragmatic movements for respiratory triggering.

Copyright (c) 2018 Wolters Kluwer Health, Inc. Unauthorized reproduction of this article is prohibited. This paper can be cited using the date of access and the unique DOI number which can be found in the footnotes. 
An example of the planning process on coronal scout images for both groups is presented in Figure 1.

\section{Evaluation of the Planning Process and MRI Examination Time}

To monitor the planning process on the scanner, the whole planning screen for all sequences was recorded by using Camtasia (TechSmith Corporation, Okemos, MI) in both groups. One medical school student analyzed these videos counting the number of user interactions and the duration of the planning process and the whole MRI examination. Mouse clicks for each confirmation in the planning process, setting of the planning boxes, confirmation after contrast media injection, adaption of sequence parameters, and each zooming or scrolling process, necessary for the planning, were counted as 1 user interaction. These user interactions were analyzed during the planning process and during the rest of the MRI examination separately. User interactions to check the acquired sequences were not counted. The planning time was defined as the time between the initial loading of the imaging protocol and the start of the first imaging sequence after the localizer sequence. The scan time was defined as the time between the start of the first imaging sequence and the end of the last imaging sequence. The whole MRI examination time was defined as the time between initial loading of the imaging protocol and the end of the last imaging sequence acquisition.

The technicians conducting the MRI examinations, who all regularly performed both types of protocols, rated the required effort for the planning process on a scale from 1 to 10 (1 meaning very low planning effort, 10 meaning very high planning effort). Furthermore, the technicians evaluated their overall preference of the protocol usage. All technicians were familiar with both scan workflows due to their daily working routine and were not specifically trained for study purposes.

\section{Analysis of Image Quality and Integrity of Study Volume}

Two radiologists with 10 and 3 years of experience in computed tomography and MRI independently reviewed all WB-MRI examinations of both groups in random order and blinded to the group type. Overall IQ and artifacts, anatomically compartmentalized into 5 regions (thorax, abdomen, pelvis, upper leg and lower leg), were assessed on a 5-point Likert scale (IQ: 1, poor; 2, below average; 3, average; 4, above average; 5, excellent; artifacts: 1 , no artifacts; 2 , mild artifacts; 3 , moderate artifacts; 4, severe artifacts; 5, not diagnostic). Furthermore, the coverage of the study volume was assessed, again anatomically compartmentalized into 5 regions. A completely covered study volume was defined as depiction of the entire musculature from the thorax (shoulder had to be fully included) to the ankles.

\section{Analysis of Patient Comfort}

After the MRI examination, patient comfort was evaluated with a questionnaire. It had 5 questions, each with a colored visual analogue scale from 0 to 10 allowing each patient to indicate the degree of (1) comfort during being positioned on the MR table, (2) available space in the MR scanner, (3) comfort of the surface coils, (4) significance of noise, and (5) length of the examination. Throughout, zero corresponds to the worst score and 10 to the best score.

\section{Statistical Analysis}

Quantitative results are reported as mean \pm standard deviation (SD) and ranges. Interreader agreement for all image-quality features, artifacts, and completeness of examination volume was assessed using the intraclass correlation coefficient (ICC). An ICC of 0.75 to 1.00 indicated excellent; 0.60 to 0.74 , good; 0.40 to 0.59 , fair; and less than 0.4 , poor agreement. ${ }^{16}$ Mann-Whitney $U$ tests were used to assess differences in patient demographics, overall IQ, artifacts, and incomplete study volume as well as differences in number of user interactions, examination, and planning duration. Furthermore, differences in the technicians' preference and in patients' comfort between group A and B were also calculated with Mann-Whitney $U$ tests.

All statistical analyses were performed using SPSS (IBM SPSS Statistics 22; SPSS Inc, Chicago IL) and Excel software (Microsoft Corporation, Redmond, WA). $P$ less than 0.05 was considered statistically significant.

\section{RESULTS}

\section{Study Population}

Patient demographics are summarized in Table 1. There were no significant differences regarding sex, weight, height, and BMI $(P=0.648, P=0.820, P=0.705$, and $P=0.623$, respectively $)$. Patients in group A were significantly older than patients in group $\mathrm{B}(P=0.017)$. There was no significant difference in the presence of visible pathology between both groups ( 2 of 10 patients in each group; $P=1$ ).

\section{Planning Process and MRI Examination}

On average, the number of user interactions was significantly lower for both the planning process $(10.2 \pm 4.4$; range, 7-22; $P<0.0001)$ and the rest of the MRI examination $(6.9 \pm 1.9$; range, 3-10; $P<0.0001)$ using the automated workflow compared with the manual workflow (48.2 \pm 17.2 ; range, 32-92; $14.9 \pm 3.1$; range, 12-21) (Fig. 2). The duration of the planning process and the duration of the whole MRI examination were significantly reduced using the automated workflow $(P<0.0001$ for both) with a mean duration of $2.5 \pm 0.8$ minutes (range, 1.9-4.2 minutes) for the planning process

TABLE 1. Patient Demographics

\begin{tabular}{|c|c|c|c|}
\hline & $\begin{array}{c}\text { Group A } \\
\text { (Automatic Workflow) }\end{array}$ & $\begin{array}{c}\text { Group B } \\
\text { (Manual Workflow) }\end{array}$ & $P$ \\
\hline Sex, female/male & $7 / 3$ & $6 / 4$ & 0.648 \\
\hline Age, y & $\begin{array}{l}52.2 \pm 13.2 \\
\quad(28-67)\end{array}$ & $\begin{array}{l}35.9 \pm 12.4 \\
\quad(21-52)\end{array}$ & 0.017 \\
\hline Weight, kg & $\begin{array}{l}69.9 \pm 13.3 \\
\quad(50-90)\end{array}$ & $\begin{array}{c}72.0 \pm 21.2 \\
(54-115)\end{array}$ & 0.820 \\
\hline Height, cm & $\begin{array}{c}173.0 \pm 9.3 \\
(163-189)\end{array}$ & $\begin{array}{c}169.2 \pm 10.3 \\
(156-189)\end{array}$ & 0.705 \\
\hline BMI, $\mathrm{kg} / \mathrm{m}^{2}$ & $\begin{array}{l}23.2 \pm 3.0 \\
(18-28)\end{array}$ & $\begin{array}{c}24.9 \pm 5.6 \\
(19-39)\end{array}$ & 0.623 \\
\hline
\end{tabular}

Comparison of group A and B using the Mann-Whitney $U$ test. 


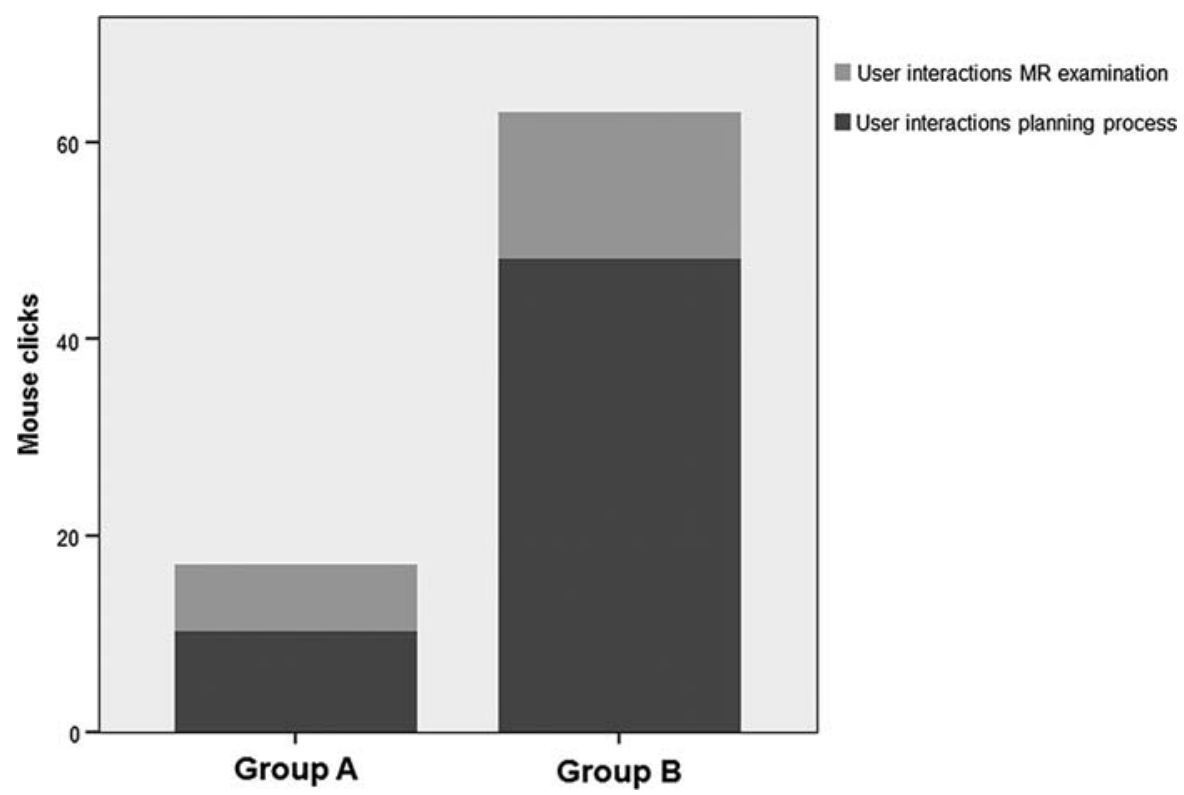

FIGURE 2. Required user interactions for the planning process (dark gray) and for the rest of the MRI examination (bright gray) for the automated (group A) and the manual WB-MRI workflow (group B). The number of user interactions was significantly lower for the automated workflow compared with the manual workflow for the planning process (mean $10.2 \pm 4.4$ vs $48.2 \pm 17.2, P<0.0001$ ) and the rest of the MRI examination (mean $6.9 \pm 1.9$ vs $14.9 \pm 3.1, P<0.0001)$.

and $30.0 \pm 4.2$ minutes (range, 26.6-39.3 minutes) for the whole MRI examination, compared with the manual workflow with $14.0 \pm 7.0$ minutes (range, 4.0-25.0 minutes) planning time and 41.5 \pm 3.4 minutes (range, 35.4 46.1 minutes) for the whole MRI examination. Thus, the automation allowed a shortening of the planning time by $82.3 \%$ and a shortening of the total examination time by $27.8 \%$. The planning took $8 \%$ of the total examination time with the automated workflow and $34 \%$ with the manual workflow $(P<0.0001)$. The scan time was almost the same in both groups $(P=0.940)$ with a mean duration of $27.5 \pm 4.3$ minutes (range, 22.9-37.2 minutes) for the automated workflow and $27.5 \pm 7.1$ minutes (range, 15.9-36.8 minutes) for the manual workflow. A comparison of planning and scan time between both groups is presented in Figure 3.

Eight technicians performed the MRI examinations. When the technicians were asked about their preference concerning the WB-MRI workflow, 7 (88\%) preferred the automated workflow. The planning effort for the MR technicians was rated significantly lower for the

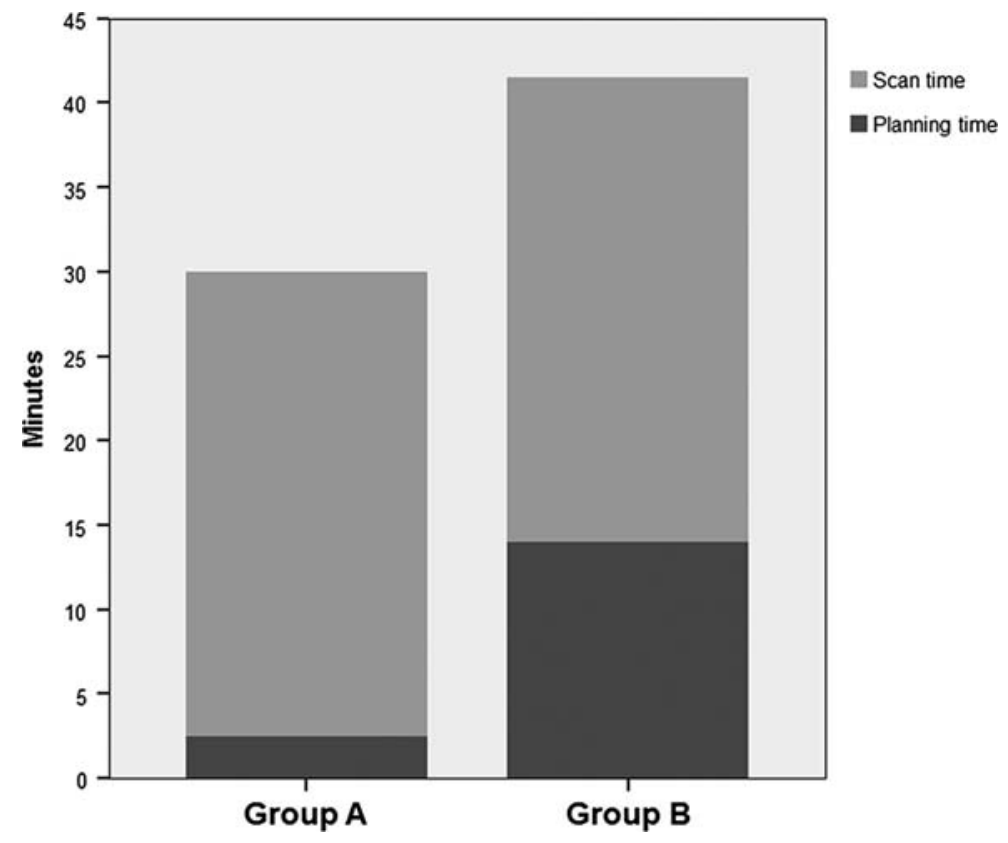

FIGURE 3. Required time for the planning process (dark gray) and for the scan of the MRI examination (bright gray) for the automated (group A) and the manual WB-MIR workflow (group B). The duration was significantly lower for the automated workflow compared with the manual workflow for the planning process (mean $2.5 \pm 0.8$ minutes vs $14.0 \pm 7.0$ minutes, $P<0.0001$ ) and the rest of the MRI examination (mean $30.0 \pm 4.2$ minutes vs $41.5 \pm 3.4$ minutes, $P<0.0001$ ). The scan time was almost the same in both groups (mean $27.5 \pm 4.3$ minutes vs $27.5 \pm 7.1$ minutes, $P=0.940$ ). 
TABLE 2. Results for the Reading of Artifacts and Overall Image Quality

\begin{tabular}{|c|c|c|c|c|c|c|c|c|c|c|}
\hline \multirow{2}{*}{$\begin{array}{l}\text { Anatomical } \\
\text { Compartment }\end{array}$} & \multicolumn{2}{|c|}{ TIRM Automatic } & \multicolumn{2}{|c|}{ TIRM Manual } & \multirow[b]{2}{*}{$P($ R1/R2) } & \multicolumn{2}{|c|}{ ce-T1w Automatic } & \multicolumn{2}{|c|}{ ce-T1w Manual } & \multirow[b]{2}{*}{$P(\mathrm{R} 1 / \mathrm{R} 2)$} \\
\hline & $\mathbf{R} 1$ & $\mathbf{R 2}$ & R1 & $\mathbf{R 2}$ & & R1 & $\mathbf{R 2}$ & R1 & $\mathbf{R 2}$ & \\
\hline Thorax & $3.8 \pm 1.3(1-5)$ & $3.8 \pm 1.3(1-5)$ & $3.2 \pm 1.0(2-5)$ & $2.8 \pm 1.3(1-5)$ & $0.168 / 0.111$ & $3.9 \pm 1.1(2-5)$ & $4.0 \pm 1.2(2-5)$ & $4.6 \pm 0.7(3-5)$ & $4.3 \pm 0.8(3-5)$ & $0.090 / 0.626$ \\
\hline Abdomen & $4.2 \pm 0.8(3-5)$ & $4.3 \pm 0.7(3-5)$ & $4.0 \pm 0.7(3-5)$ & $4.0 \pm 1.1(2-5)$ & $0.510 / 0.598$ & $4.5 \pm 0.5(4-5)$ & $4.6 \pm 0.5$ & $4.6 \pm 0.7(3-5)$ & $4.6 \pm 0.5(4-5)$ & $0.511 / 1$ \\
\hline Pelvic & $3.8 \pm 0.9(2-5)$ & $3.9 \pm 0.9(2-5)$ & $3.7 \pm 0.8(3-5)$ & $3.6 \pm 1.4(2-5)$ & $0.659 / 0.723$ & $4.1 \pm 1.0(2-5)$ & $3.8 \pm 0.9(2-5)$ & $4.6 \pm 0.5$ & $4.8 \pm 0.4(4-5)$ & $0.240 / 0.006$ \\
\hline Upper leg & $4.2 \pm 0.6(3-5)$ & $4.0 \pm 0.7(3-5)$ & $4.2 \pm 0.8(3-5)$ & $3.8 \pm 1.0(2-5)$ & $0.934 / 0.687$ & $4.3 \pm 0.9(2-5)$ & $4.5 \pm 0.7(3-5)$ & $4.7 \pm 0.5$ & $4.9 \pm 0.3(4-5)$ & $0.313 / 0.121$ \\
\hline Lower leg & $4.3 \pm 0.5(4-5)$ & $4.5 \pm 0.5(4-5)$ & $4.0 \pm 0.9(2-5)$ & $4.1 \pm 1.1$ & $0.544 / 0.534$ & $4.4 \pm 1.3(1-5)$ & $4.4 \pm 1.3$ & $4.9 \pm 0.3(4-5)$ & $4.9 \pm 0.3(4-5)$ & $0.255 / 0.255$ \\
\hline Overall IQ & $4.0 \pm 1.1(2-5)$ & $4.0 \pm 0.9(2-5)$ & $3.5 \pm 1.2(2-5)$ & $3.4 \pm 1.3(2-5)$ & $0.325 / 0.273$ & $4.2 \pm 1.0(2-5)$ & $4.2 \pm 0.8$ & $4.5 \pm 0.7(3-5)$ & $4.6 \pm 0.5(4-5)$ & $0.556 / 0.240$ \\
\hline
\end{tabular}

Results are presented as mean \pm standard deviation and the range in parentheses. The $P$ value was evaluated between the automated and manual WB-MRI workflow for both readers using the Mann-Whitney $U$ test.

TIRM indicates turbo inversion recovery magnitude; ce-T1w, contrast-enhanced T1-weighted Dixon; R1, reader 1; R2, reader 2; IQ, image quality.

automated workflow compared with the manual workflow $(2.2 \pm 0.9$, range $1-3$ and $4.8 \pm 2.4$, range $1-9$, respectively; $P=0.005)$.

\section{Image Quality and Integrity of Study Volume}

There were no significant differences between groups A and B in overall IQ for the TIRM (reader $1 / 2: P=0.325 / 0.273$ ) and the ce-T1w sequence (reader 1/2: $P=0.556 / 0.240$ ) for both readers. Significantly, more artifacts were seen by reader 2 in the ce-T1w sequence in the pelvic compartment of group B compared with group A (mean score, $4.8 \pm 0.4$ for group B vs $3.8 \pm 0.9$ for group $\mathrm{A} ; P=0.006$ ). In all other anatomic compartments, there were no significant differences for artifacts between automated and manual workflow for both readers $(P=0.090$ to $P=1.0)$. Results for the reading of artifacts and overall IQ are summarized in Table 2. An example of the coronal TIRM sequence of both groups is presented side by side in Figure 4.

Incomplete coverage in the TIRM sequence occurred in the thoracic compartment in $60 \%$ (shoulders incomplete, 6 of 10, same for both readers) of patients in groups A and B. In 10\% (1 of 10, same for both readers) of the patients, the study volume of the pelvic compartment was incomplete in the TIRM sequence in group B. All other anatomical regions were completely covered using the TIRM sequence. In the ceT1w sequence, incomplete coverage of the study volume occurred in the thoracic compartment (shoulders incomplete) in 60\% (6 of 10) and $70 \%$ (7 of 10) in group A and in 30\% (3 of 10) and 10\% (1 of 10) in group $\mathrm{B}$ for reader 1 and 2, respectively. Reader 2 assessed all other anatomical compartments as completely covered. Reader 1 assessed the abdominal, pelvic, and upper and lower leg compartments on the ce-T1w sequence as incomplete in $20 \%$ in group B and the lower leg compartment in $10 \%$ ( 1 of 10 ) in group A. The study volume of the thoracic compartment in the ceT1w sequence was significantly more often incompletely covered in group A than in group B $(P=0.008)$ for reader 2 . No other significant differences in the coverage of the study volume were found between the both groups.

Interreader agreement was excellent for overall IQ with an ICC of 0.95 (95\% confidence interval [CI], 0.86-0.98) for the TIRM sequence and $0.88(95 \% \mathrm{CI}, 0.70-0.95)$ for the ce-T1w sequence. Interreader agreement was poor for artifacts in the ce-T1w sequence of the abdominal region (ICC, $0.39 ; 95 \% \mathrm{CI},-0.54$ to 0.76 ) and good for artifacts in the ce-T1w sequence of the upper leg (ICC, $0.64 ; 95 \%$ CI, 0.08-0.86). Furthermore, interreader agreement for the coverage of the examination volume in the TIRM sequences for the thoracic region was good (ICC, 0.74; 95\% CI, 0.34-0.90). For all other parameters, including artifacts and coverage of the study volume in both sequences, interreader agreement was excellent (ICC, 0.77-1).

\section{Patient Comfort}

Results of patient comfort are summarized in Table 3. There were no significant differences between groups A and B concerning the comfort of positioning on the MR table, space in the MR scanner, comfort of the placed surface coils, the noise during the MRI examination, and the length of the whole examination.

\section{DISCUSSION}

In our study, the automated WB-MRI workflow significantly reduced the examination time and the number of operator interactions during the planning process and during the whole MRI examination compared with the manual WB-MRI workflow. Image quality was equivalent for both groups and the MR technicians' acceptance was higher for the automated workflow.

Our study results are a successful example for a data acquisition workflow improvement representative of automation and optimization in radiology with the ultimate goal of increased time efficiency. So far, the main workflow optimization processes have focused on the handling of acquired images with complete digitalization in radiology departments and the direct linkage of radiology information system with the picture archiving and communication system ${ }^{17-19}$ or have focused on the radiologists reading process. ${ }^{20}$ The on-going automation of the scanning workflow may reduce the time needed for sequence planning before and between the individual scanning steps and lead to an increase in time efficiency of the MR scanner. The Dot (day optimizing throughput) engine (Siemens Healthcare, Erlangen, Germany) is an automated MR scanner workflow, optimized for various body parts. In our study, we used a prototype implementation of a WB-MRI Dot Engine. The goal is a reduction of the number of user interactions and of examination time variations between patients. A previous study showed a significant reduction of total examination time and necessity for technician interventions by using this automated workflow application in MRI head examinations. ${ }^{15}$ There, the total examination time was reduced by $20 \%$ ( 24 minutes 53 seconds vs 20 minutes 1 second), which was less compared with our WB-MRI workflow study with a total examination time reduction of $27.8 \%$ (41 minutes 30 seconds vs 29 minutes 59 seconds). It can be concluded that MRI examinations covering multiple body parts such as a WB-MRI benefit even more from such an automated workflow in terms of whole examination time reduction than a single station MRI examination.

When looking at the MRI scanner workflow with the Lean Six Sigma method, ${ }^{21} 3$ classifications of time can be differentiated: $(a)$ value-added time, meaning time with direct benefit to the patient when actual image acquisition takes place, $(b)$ business value-added time, time to perform technically required steps on the MRI scanner (ie, prescan adjustments, localizer), but which would preferably be avoided, and $(c)$ non-value-added time, meaning time when the hardware is inactive waiting for operator instructions. In this approach of increasing time efficiency in the WB-MRI scanner workflow, it would be preferable to first minimize non-value-added time, second business 


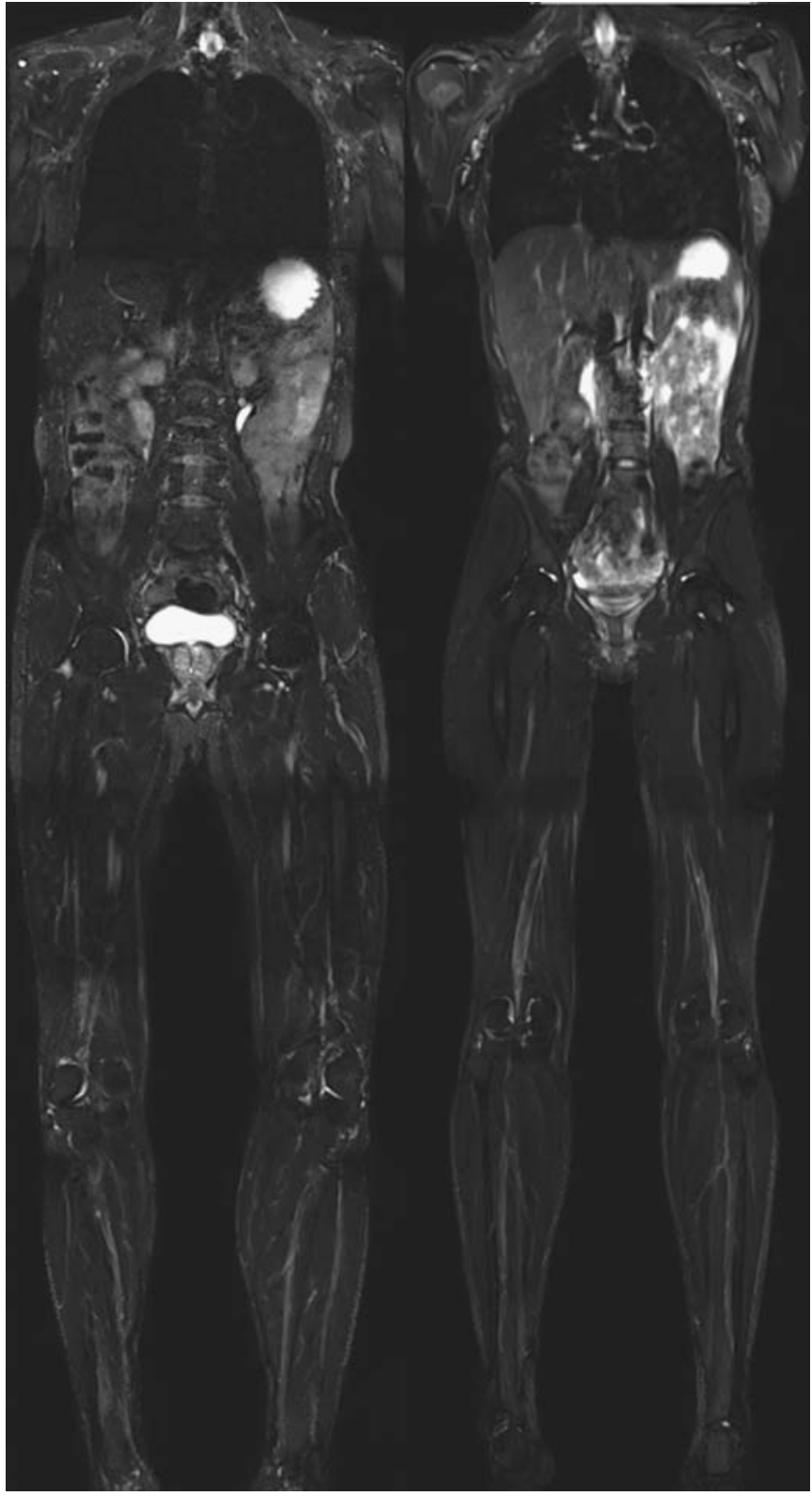

FIGURE 4. Example of a coronal TIRM sequence of the automated WB-MRI workflow (left side) and the manual WB-MRI workflow (right side) showing comparable image quality.

value-added time, and third value-added time. The proposed automated WB-MRI scanner workflow reduces the non-value-added time by reducing the number of user interactions and thus the time the scanner is inactive, and it also reduces the business value-added time by reducing the time spent on localizer imaging using a fast low-resolution moving table whole-body localizer sequence resulting in a time reduction of $85 \%$ of these 2 components.

In another approach to reduce the business value-added time between actual image acquisitions, the extent of prescan adjustments, such as field homogenization ("shimming"), or frequency optimization, between sequences has been reduced to a minimum. Bashir et $\mathrm{al}^{22}$ reduced the business value-added time in noncontrast liver MRI by $58 \%$ and the total table time by $20 \%$ ( 22 minutes 48 seconds vs 18 minutes 13 seconds) by elimination of table movement and, thus, reduced shimming adjustments. For our WB-MRI protocol using the manual workflow, scanning with a fixed table position would not be possible. Furthermore, the influence of a reduction of shimming adjustment on the IQ remains unclear as TIRM and Dixon sequences are insensitive to B0 inhomogeneity and shimming might not be necessary anyway in these sequences.

Other approaches to the reduction of classified time segments have been attempts to reduce the actual image acquisition time (valueadded time) with new image acquisition techniques. A very successful example of image acquisition time reduction is the parallel imaging technique. $^{23}$ Another example is the recently developed simultaneous multislice acceleration technique, which allows up to 4-fold accelerated image acquisition at the expense of a certain degree of IQ reduction with high acceleration factors. ${ }^{24,25}$ In a whole-body diffusion-weighted echo-planar imaging protocol, the image acquisition time of the diffusion-weighted imaging sequence with an acceleration factor of 3 could be reduced by $26 \%{ }^{26}$ In a study of diffusion-tensor imaging (DTI) of the muscles of the lower leg, the simultaneous multislice acceleration technique allowed an image acquisition time reduction of $48 \%$ compared with conventional, nonaccelerated DTI ( 7 minutes $24 \mathrm{sec}-$ onds vs 3 minutes 53 seconds). ${ }^{27}$ Although DTI is not part of our standard MRI protocol for the assessment of myopathies, it proved to be a feasible method for the detection of muscle changes ${ }^{28,29}$ and may be an important part of future WB-MRI protocols. Another rapidly emerging technique for rapid image acquisition is compressed sensing, which recently has become commercially available by several vendors. Compressed sensing is based on a mathematical framework, which uses highly undersampled data and incompletely filled k-space to reconstruct the images. $^{30,31}$ Yoon et $\mathrm{al}^{32}$ previously demonstrated even a time reduction of up to $96 \%$ ( 7 minutes vs 16 seconds) in 3D magnetic resonance cholangiopancreatography by using compressed sensing with comparable IQ.

These approaches that reduce actual image acquisition time by optimizing acquisition techniques while still striving to preserve high IQ seem to have been very successful, with variable effects on the individual process step with relative time reductions between one and even three thirds of imaging time. A higher potential in time reduction of an individual process step can be seen after workflow optimization with $85 \%$ time reduction of the business value-added and non-value-added time in our study.

With regard to the total MRI examination time, the effect of omitting image acquisition steps seems to be comparable to workflow optimization in WB-MRI protocols. ${ }^{27}$ A reduction of $32 \%$ may be possible in a protocol using the same sequences as our WB-MRI protocol, when omitting to scan chest and abdomen compared with our total MRI examination time reduction of $28 \%$ after workflow automation. However, the $32 \%$ time reduction from 56 to 37 minutes' scan time is only an approximation, because no exact imaging times where recorded in this study. ${ }^{27}$ Furthermore, dropping imaging of body parts has to be evaluated with caution so that important findings are not missed. ${ }^{14}$ In our study, we could cover the whole body on average within 29 minutes 59 seconds with our automated WB-MRI workflow without lacking trunk information. Besides imaging patients with myopathy, this automated WB-MRI workflow can be used for multiple indications affecting the whole body or for systemic diseases. For example, WB-MRI showed to be a promising tool in oncologic imaging for the staging and the assessment of therapeutic response in various oncological diseases. ${ }^{33}$

In our study, the overall IQ was comparable between groups A and $\mathrm{B}$. The TIRM sequences showed a tendency toward slightly higher overall IQ in group A, while the ce-T1w sequences showed the opposite trend. Artifacts were less severe in patients in group A in the TIRM sequences and more severe in the ce-T1w sequences compared with group B. Significant differences concerning artifacts were only detected in the pelvic compartment in the ce-T1w sequences for reader 2 with more severe artifacts in group B. More important than the minimal differences in IQ is the coverage of the study volume as this is directly 
TABLE 3. Results of Patients' Comfort Survey

\begin{tabular}{|c|c|c|c|c|}
\hline & Issue & Group A & Group B & $\boldsymbol{P}$ \\
\hline Q1 & Comfort of positioning on MR table & $\begin{array}{c}7.1 \pm 1.7 \\
(4-10)\end{array}$ & $\begin{array}{c}8.0 \pm 1.6 \\
(5-10)\end{array}$ & 0.232 \\
\hline Q2 & Space in the MR scanner & $\begin{array}{c}7.7 \pm 1.7 \\
(4-10)\end{array}$ & $\begin{array}{c}7.6 \pm 2.5 \\
(2-10)\end{array}$ & 0.877 \\
\hline Q3 & Comfort of the surface coils & $\begin{array}{c}3.5 \pm 1.4 \\
(1-6)\end{array}$ & $\begin{array}{c}4.6 \pm 2.7 \\
(2-10)\end{array}$ & 0.396 \\
\hline Q4 & Insignificance of noise during examination & $\begin{array}{c}5.2 \pm 2.2 \\
(1-8)\end{array}$ & $\begin{array}{c}5.2 \pm 1.9 \\
(2-8)\end{array}$ & 0.969 \\
\hline Q5 & Length of the MRI examination & $\begin{array}{c}6.8 \pm 1.8 \\
(4-10)\end{array}$ & $\begin{array}{c}6.3 \pm 2.2 \\
(3-10)\end{array}$ & 0.592 \\
\hline
\end{tabular}

Results of patients' comfort survey (mean \pm standard deviation and the range in parentheses, group A [automated WB-MRI workflow] and group B [manual WB-MRI workflow]) were compared using the Mann-Whitney $U$ test.

linked to the planning process on the scanner. An incomplete coverage was most often seen in the thoracic compartment in the TIRM and the ce-T1w sequences in both groups, meaning that the muscle groups of the shoulders were not completely covered. This circumstance can be explained by an inaccurate planning process by the technicians. In both the automated and the manual workflow, the complete coverage of the volume of interest had to be determined and approved by the technician to fully cover the important muscle groups. We assume that this problem is not related to any technical aspect of the sequences or scanner workflow and could be solved by giving clear instructions for the determination of the volume of interest and by improving the technicians' training of the planning process.

The technicians clearly favored the automated workflow over the manual workflow. This is attributable to the significantly less intense user interaction required for the planning process and the rest of the MRI examination. A reduction of user interaction and a higher acceptance by the technicians, because of a simplified workflow, reduces the susceptibility to errors. Especially for technicians who are inexperienced in WB-MRI, due to few WB-MRI examinations in their institute or their educational level, an automated workflow might be beneficial.

Our study showed no influence of the reduced total examination time when scanning with the automated workflow on the patients' perception of comfort in the scanner. The duration of the MRI scan was not perceived differently in both groups, which may be related to their missing reference to MRI examination times in general. All other factors (comfort on the table, space in the scanner, surface coils, and noise), which might influence the patients' well-being in the scanner, were the same during both workflows and were also not differently perceived in the 2 patient groups. However, long examination times might negatively influence the patients' comfort and compliance and, therefore, should be kept as short as possible. ${ }^{34}$

The following study limitations must be taken into account. First, the sample size of 20 patients was small; however, the statistical power for the main parameters was sufficient. Second, pathological findings were found in only $10 \%$ of the patients; therefore, the influence of scan duration on patient comfort may have been underestimated, and patients with myopathy may experience more discomfort during a longer MRI examination. Furthermore, unlikely differences in the validity of the 2 scanner workflows for pathologic pattern recognition have not been evaluated. Third, 2 different patient cohorts were subject to the 2 MRI protocols with the possibility of selection bias. However, there were no significant differences in sex, weight, height, and BMI between group $\mathrm{A}$ and $\mathrm{B}$; therefore, the influence of the patients' demographics on the study result is negligible.

In conclusion, the automated WB-MRI scanner workflow showed a significant reduction of the examination time and the user interaction compared with the manual WB-MRI workflow. Image quality and the coverage of the study volume were comparable in both groups. The automated WB-MRI scanner workflow would also be useful for oncological WB-MRI indications and should be studied further.

\section{REFERENCES}

1. Bernatsky S, Joseph L, Pineau CA, et al. Estimating the prevalence of polymyositis and dermatomyositis from administrative data: age, sex and regional differences. Ann Rheum Dis. 2009;68:1192-1196.

2. Dimachkie MM, Barohn RJ, Amato AA. Idiopathic inflammatory myopathies. Neurol Clin. 2014;32:595-628. vii.

3. El-Tallawy HN, Khedr EM, Qayed MH, et al. Epidemiological study of muscular disorders in Assiut, Egypt. Neuroepidemiology. 2005;25:205-211.

4. Meyer A, Meyer N, Schaeffer M, et al. Incidence and prevalence of inflammatory myopathies: a systematic review. Rheumatology (Oxford). 2015;54:50-63.

5. Cantwell C, Ryan M, O'Connell M, et al. A comparison of inflammatory myopathies at whole-body turbo STIR MRI. Clin Radiol. 2005;60:261-267.

6. Elessawy SS, Abdelsalam EM, Abdel Razek E. Whole-body MRI for full assessment and characterization of diffuse inflammatory myopathy. Acta Radiol Open. 2016;5:2058460116668216

7. O'Connell MJ, Powell T, Brennan D, et al. Whole-body MR imaging in the diagnosis of polymyositis. AJR Am J Roentgenol. 2002;179:967-971.

8. Schmidt GP, Reiser MF, Baur-Melnyk A. Whole-body imaging of the musculoskeletal system: the value of MR imaging. Skeletal Radiol. 2007;36:1109-1119.

9. Yosipovitch G, Beniaminov O, Rousso I, et al. STIR magnetic resonance imaging: a noninvasive method for detection and follow-up of dermatomyositis. Arch Dermatol. 1999; 135:721-723.

10. Filli L, Maurer B, Manoliu A, et al. Whole-body MRI in adult inflammatory myopathies: do we need imaging of the trunk? Eur Radiol. 2015;25:3499-3507.

11. Lenk S, Fischer S, Kotter I, et al. Possibilities of whole-body MRI for investigating musculoskeletal diseases [in German]. Radiologe. 2004;44:844-853.

12. Dalakas MC. Inflammatory muscle diseases. N Engl J Med. 2015;373:393-394.

13. Day J, Patel S, Limaye V. The role of magnetic resonance imaging techniques in evaluation and management of the idiopathic inflammatory myopathies. Semin Arthritis Rheum. 2017;46:642-649.

14. Malattia C, Damasio MB, Madeo A, et al. Whole-body MRI in the assessment of disease activity in juvenile dermatomyositis. Ann Rheum Dis. 2014;73 1083-1090.

15. Moenninghoff C, Umutlu L, Kloeters C, et al. Workflow efficiency of two $1.5 \mathrm{~T}$ MR scanners with and without an automated user interface for head examinations. Acad Radiol. 2013;20:721-730.

16. Landis JR, Koch GG. The measurement of observer agreement for categorical data. Biometrics. 1977;33:159-174.

17. Faggioni L, Neri E, Cerri F, et al. Integrating image processing in PACS. Eur J Radiol. 2011;78:210-224.

18. Langen HL, Bielmeier J, Wittenberg G, et al. Workflow improvement and efficiency gain with near total digitalization of a radiology department [in German]. Rofo. 2003;175:1309-1316.

19. Redfern RO, Langlotz CP, Abbuhl SB, et al. The effect of PACS on the time required for technologists to produce radiographic images in the emergency department radiology suite. J Digit Imaging. 2002;15:153-160. 
20. Reiner B. New strategies for medical data mining, part 3: automated workflow analysis and optimization. J Am Coll Radiol. 2011;8:132-138.

21. Roth CJ, Boll DT, Wall LK, et al. Evaluation of MRI acquisition workflow with lean six sigma method: case study of liver and knee examinations. AJR Am J Roentgenol. 2010;195:W150-W156.

22. Bashir MR, Dale BM, Gupta RT, et al. Gradient shimming during magnetic resonance imaging of the liver: comparison of a standard protocol versus a novel reduced protocol. Invest Radiol. 2012;47:524-529.

23. Pruessmann KP, Weiger M, Scheidegger MB, et al. SENSE: sensitivity encoding for fast MRI. Magn Reson Med. 1999;42:952-962.

24. Boss A, Barth B, Filli L, et al. Simultaneous multi-slice echo planar diffusion weighted imaging of the liver and the pancreas: optimization of signal-to-noise ratio and acquisition time and application to intravoxel incoherent motion analysis. Eur J Radiol. 2016;85:1948-1955.

25. Kenkel D, Barth BK, Piccirelli M, et al. Simultaneous multislice diffusionweighted imaging of the kidney: a systematic analysis of image quality. Invest Radiol. 2017;52:163-169.

26. Kenkel D, Wurnig MC, Filli L, et al. Whole-body diffusion imaging applying simultaneous multi-slice excitation. Rofo. 2016;188:E1.

27. Filli L, Piccirelli M, Kenkel D, et al. Simultaneous multislice echo planar imaging with blipped controlled aliasing in parallel imaging results in higher acceleration: a promising technique for accelerated diffusion tensor imaging of skeletal muscle. Invest Radiol. 2015;50:456-463.

28. Froeling M, Oudeman J, Strijkers GJ, et al. Muscle changes detected with diffusion-tensor imaging after long-distance running. Radiology. 2015;274: 548-562.

29. Zaraiskaya T, Kumbhare D, Noseworthy MD. Diffusion tensor imaging in evaluation of human skeletal muscle injury. J Magn Reson Imaging. 2006;24:402-408

30. Sharma SD, Fong CL, Tzung BS, et al. Clinical image quality assessment of accelerated magnetic resonance neuroimaging using compressed sensing. Invest Radiol. 2013;48:638-645.

31. Worters PW, Sung K, Stevens KJ, et al. Compressed-sensing multispectral imaging of the postoperative spine. J Magn Reson Imaging. 2013;37:243-248.

32. Yoon JH, Lee SM, Kang HJ, et al. Clinical feasibility of 3-dimensional magnetic resonance cholangiopancreatography using compressed sensing: comparison of image quality and diagnostic performance. Invest Radiol. 2017;52:612-619.

33. Morone M, Bali MA, Tunariu N, et al. Whole-body MRI: current applications in oncology. AJR Am J Roentgenol. 2017;209:W336-W349.

34. Guckel B, Gatidis S, Enck P, et al. Patient comfort during positron emission tomography/magnetic resonance and positron emission tomography/computed tomography examinations: subjective assessments with visual analog scales. Invest Radiol. 2015;50:726-732. 